\title{
A Spiral Development Model for an Advanced Traffic Management System (ATMS) Architecture Based on Prototype
}

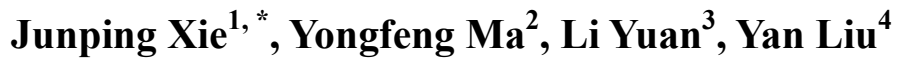 \\ ${ }^{1}$ School of Automotive and Traffic Engineering, Jiangsu University, Zhenjiang, Jiangsu, China \\ ${ }^{2}$ School of Transportation, Southeast University, Nanjing, Jiangsu, China \\ ${ }^{3}$ College of Civil and Transportation Engineering, Hohai University, Nanjing, Jiangsu, China \\ ${ }^{4}$ Department of English Language and Literature, Zhenjiang Watercraft College, Zhenjiang, Jiangsu, China
}

Email address:

jpxie@ujs.edu.cn (Junping Xie), mayf@seu.edu.cn (Yongfeng Ma), yuanlibox@sina.com (Li Yuan), 28115427@qq.com (Yan Liu)

\section{To cite this article:}

Junping Xie, Yongfeng Ma, Li Yuan, Yan Liu. A Spiral Development Model for an Advanced Traffic Management System (ATMS) Architecture Based on Prototype. International Journal of Science, Technology and Society. Vol. 3, No. 6, 2015, pp. 304-308.

doi: $10.11648 /$ j.ijsts.20150306.15

\begin{abstract}
As a symbol of economic growth, the deployment of an Intelligent Transportation System (ITS) became a necessity of a country, especially in a developing country. The Advanced Transportation Management System (ATMS) is one of the pivotal subsystems of ITS. With the development of digital image processing, the deployment of such a system can be implemented in an effective and economical way. The process of constructing an ATMS with minimum cost and time will be an important criterion in the selection of such a system for a developing country. In this paper, a spiral development method for an ATMS based on prototype was presented in order to overcome the shortcomings of the traditional waterfall model. The focus on the spiral development is to improve the design concepts and system requirements to avoid large-scale modification in the later development phases due to errors caused in the early ones. An applied architecture is constituted with the innovative technologies, e.g. 4th-generation mobile technology $(4 \mathrm{G})$, high-speed optical fiber communication technology with a national traffic communication backbone network in China, which ensures the latest technology is available to traffic management.
\end{abstract}

Keywords: Spiral Development Model, Prototype, ATMS, Architecture

\section{Introduction}

The problems of transportation become serious because of the rapid economic development in China. It is known that infrastructure constructions cannot solve the traffic problems well without the assistance of advanced technologies [1]. Intelligent Transportation System (ITS) can provide a real-time, accurate and efficient traffic management by integrating advanced technologies together such as information technology, electronic control technique, transducer and computer technologies. The aim of ITS is to enhance efficiency and safety of transportation, and improve the ecological environment and energy utilization efficiency greatly through making people, vehicle and road cooperative [2]. Advanced Traffic Management System (ATMS), which is an important sub-system of ITS, implements emerging computer, communication, and information technology to provide vital information to the governors of a system to raise the safety and efficiency of traffic, and to reduce the congestions and the environmental pollution [3]. Because of the vital role that ATMS plays in ITS, the implementation of an ATMS is valued by the government in China, especially in urban areas.

To develop an appropriate architecture plays an important role in constructing an ITS application. The U.S. Department of Transport (U.S. DOT) firstly published national ITS architecture in the world to provide a common framework for planning, defining, and integrating ITS. The architecture keeps improving and reaches the latest version 7.1. Based on the national ITS architecture, Mc Trans Software Center at the University of Florida has developed a software application named Turbo Architecture, which supports development of regional and project ITS architectures using the national ITS architecture as a starting point. The software is updated to version 7.1 according to national architecture [4]. In addition, 
kinds of projects of ITS and ATIS are carried on promoting the transportation development in the United States [5] [6]. On the other hand, there is a short history of researching ITS in China. The National Center of ITS Engineering and Technology (ITSC), which plays an important role in Chinese ITS researches, developed Chinese national ITS architecture in 2003, and a computer-aided ITS architecture development tool called ITSA-CASS [7]. The updated version 2 for the Chinese national architecture has not been published so far, however. Aided by the Chinese national ITS architecture, some regional ITS architectures have been developed gradually [8] [9] [10].

To construct an ITS applied architecture is very important in the design phase of implementing the ITS application with the instructions of the national ITS architecture or regional architecture. The traditional methodology of constructing the applied architecture is usually the waterfall model which is borrowed from software engineering. The waterfall model is a sequential design process, used in software development processes, in which progress is seen as flowing steadily downwards (like a waterfall) through the phases of requirements, analysis, design, coding, testing and operation (Figure 1). In an ITS application design process, the usual steps are shown in Figure 2 according to the waterfall model. The waterfall model provides a structured approach; the model itself progresses linearly through discrete, easily understandable and explainable phases and thus is easy to understand; it also provides easily identifiable milestones in the development process [11]. Although the waterfall model is seen as an agile system development method, there are more and more critical arguments about it. Designers may not know exactly what their requirements are before they see working system and so change their requirements, leading to redesign, redevelopment, and retesting, and increased costs. Designers may not be aware of future difficulties when designing a new product or feature; in which case, it is better to revise the design than keeping a design that does not account for any newly discovered constraints, requirements, or problems [12] [13]. As the conditions modify quickly in an ITS application design process, another advanced model, which is called spiral model, is more suitable than the waterfall model.

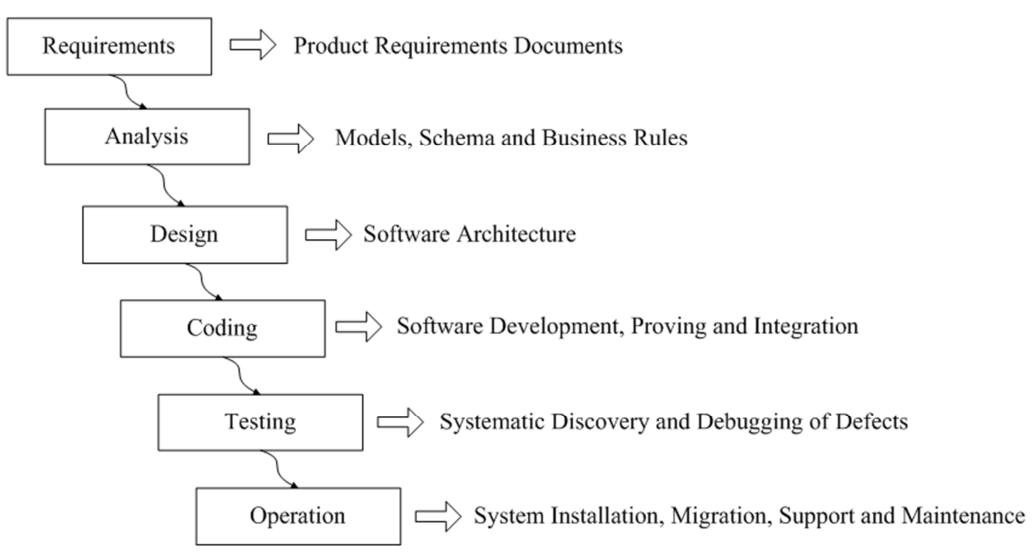

Figure 1. Traditional Waterfall Model Progress in Software Engineering.

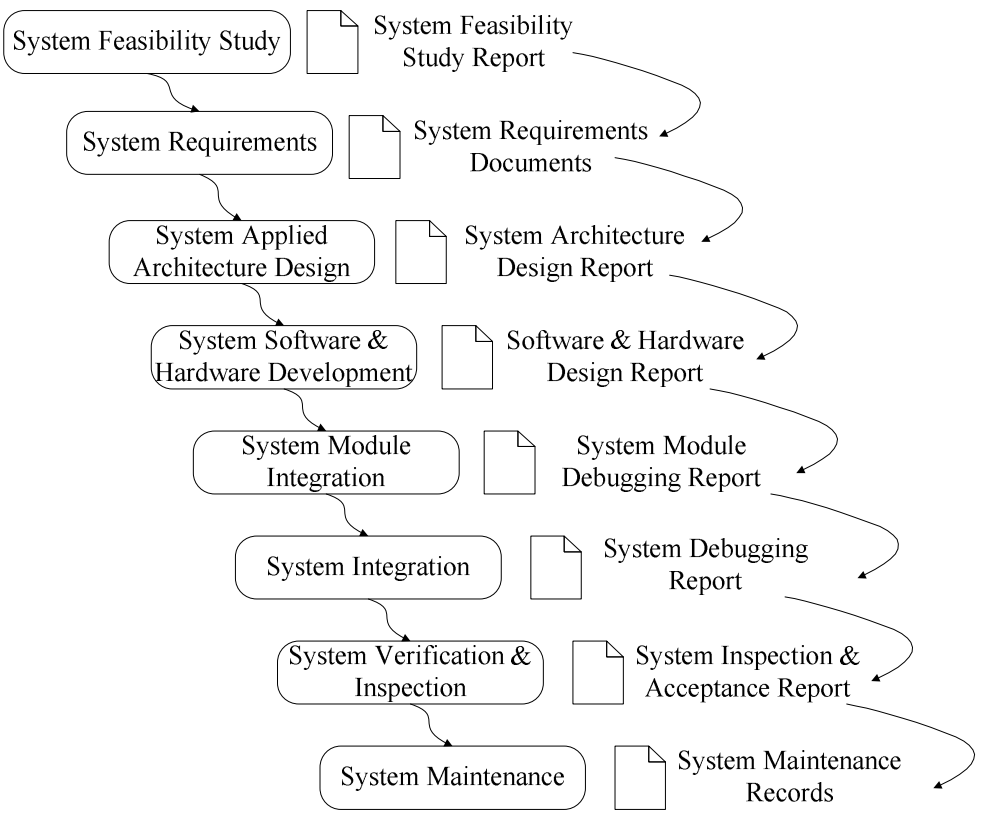

Figure 2. ITS Application System Development Model Based on Waterfall Model. 
The spiral model was first described by Boehm in his paper in 1986 [14]. In 1988, he published a similar paper to a wider audience [15]. These papers introduce a diagram that has been reproduced in many subsequent publications discussing the spiral model. The greatest advantage of the spiral model is that it allows the model to accommodate any appropriate mixture of a specification-oriented, prototype-oriented, simulation-oriented, automatic transformation-oriented or other approach to system development. This characteristic can be used in constructing ITS applications for the changeful conditions in the building process.

This paper presents the method of constructing an ATMS using a spiral development model based on prototype.

\section{Spiral Development Model for ATMS}

The spiral development model for an ATMS is combined with traditional waterfall model and prototype-oriented model Risk identification, risk analysis and risk control are brought in before each system developing phase. The following step will not be carried on until the measures to eliminate the risks in the present step are implemented.

The development model is divided into 3 circles: system concept development circle, system prototype development circle and system engineering development circle (Figure 3), which begin with the 3 requirements steps: system requirements, preliminary design requirements and detail design requirements, respectively.

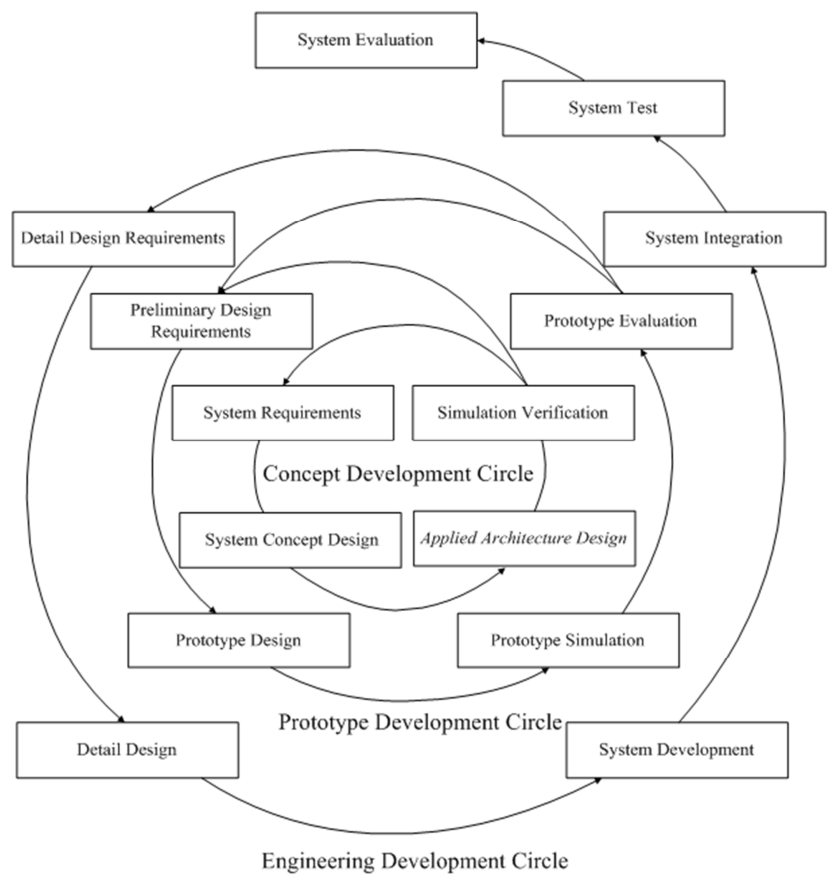

Figure 3. Spiral Development Model for an ATMS Based on Prototype.

In the system concept development circle, there are 4 steps included: system requirements, system concept design, applied architecture design and simulation verification. If the outputs of the simulation verification step are not well, the designers can also revise the system requirements step to run the concept development circle again. The applied architecture design step is very important to the concept development circle. It is built under the instructions of the national ITS architecture. In this paper, the applied architectures of ATMS are illustrated in the following section.

In the prototype development circle, the prototype of the ATMS is designed based on the outputs of the previous system concept development circle. The prototype can be developed on the prototype simulation platform, which includes hardware and software to test the characteristics of the prototype.

In the engineering development circle, if the detail design requirements are determined, the following design steps, which include detail design, system development, system integration, system test and system evaluation, will be carried on to complete the system development.

The applied architecture step also plays an important role through the whole development process. Before the system development, the simulation models can be constructed according to the applied architectures. The system performances and functions can be revised in simulation environment circularly until the users and designers are satisfied with the system design plan. The greatest advantage of simulation is that the functions of the system can be improved and revised easily on the simulation platform, as well as the real-time and dynamic performances can be tested using the prototype. With the spiral development model based on prototype, the design quality of the system can be improved and the cost can be reduced.

\section{ATMS Applied Architecture}

This section illustrates the applied architectures resulted from applied architecture design step. The applied architectures, as discussed earlier, are very important through the whole development process of the ATMS application system. In this paper, the ATMS applied architectures are constructed under consideration of Chinese national ITS architecture and the existing urban infrastructure situation in east China. The application system at present stage is built up mainly based on the intelligent camera system, which covers urban arterial roads, intersections, squares and city approach highways. The digital image processing can do a great deal to improve ATMS applications [16]. More functions and equipment can be added to the system if the financial input increases in the future.

The main component modules of an ATMS application system can be divided as follows:

Intelligent Camera System - It can record the motion graphics in the monitoring scope, trace the vehicles, capture traffic violations, and recognize license plates.

Intelligent Camera Access Network - It can be built up with 4G mobile technology.

Intelligent Camera Server - It acts as a camera system server if the camera nodes access the other nodes of the modules in ATMS. It also manages the intelligent camera system. 
ATMS Communication System - The communication of cameras on field can be established with $4 \mathrm{G}$ gateways, and the other communications can be accomplished via the national traffic communication backbone network in China.

ATMS Management Center - It is a center constituted with many modules such as ATMS database, expert referring system, message and web servers. The management center plays a vital role in the ATMS application system.

ATMS Payment Gateways - The real time and offline payments can be processed, and the system can communicate with the banks for fee settlement.

The overall applied architecture of ATMS is illustrated in Figure 4.

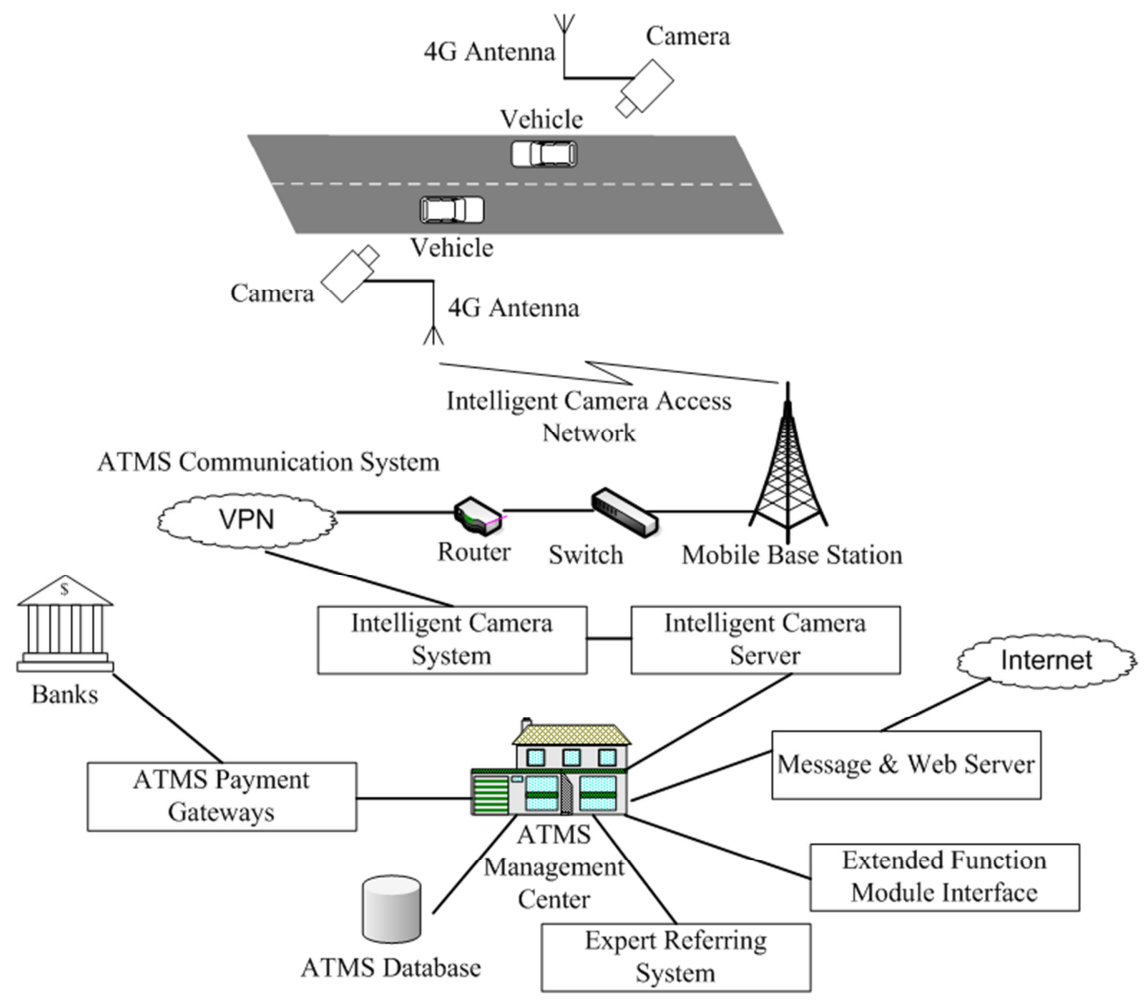

Figure 4. Overall Applied Architecture of ATMS.

\section{Conclusions}

This paper is intended to propose an innovative method for designing an ATMS using the spiral development model with prototype in China. With the development of digital image processing and other wireless communication technologies, the deployment of such a system can be implemented in an effective and economical way. The process will help the other designers to design an ATMS with less cost and time in China.

\section{Acknowledgements}

The authors would like to thank the support of the National Natural Science Foundation of China (nos.51208232, 51208100 and 51308192) and Scientific Research Foundation for Advanced Talents of Jiangsu University (No.13JDG074).

\section{References}

[1] Xie, J. P., et al. (2007). Research on Standard ATIS Design Method for Trunk Roads in China. The 10th International IEEE Conference on Intelligent Transportation Systems, Seattle, Washington, USA.
[2] Yang, B., et al. (2000). Intelligent Transportation Systems. China Railway Publishing House, Beijing, 2-4.

[3] Qiu, B. Q. (2013). Research and Design of Advanced Traffic Management System in Intelligent Transportation System. Electronic Test, (9): 107-108.

[4] U.S. DOT. (2015). National ITS Architecture Version 7.1. Retrieved June 24, 2015, from http://www.its.dot.gov/arch/index.htm.

[5] Crabtree, J. D. \& Walton J. R. (2004). Developing an Intelligent Transportation System (ITS) Architecture for the KIPDA Region (Final Report). Report No. KTC-04-23/KIPDA-02-1F.

[6] Texas DOT. (2015). Texas Regional ITS Architecture Home Page. Retrieved July 20, 2015, from http://www.consystec.com/texas/default.htm.

[7] ITSC. (2012). Introductions of Tenth Five-Year Projects. Retrieved June 10, 2015, from http://www.itsc.com.cn/ViewNews.asp?ID=7533.

[8] Lu, J., Xiang, Q. J., \& Xie, J. P. (2006). Traveler Information System Design for Network of Roads in Jiangsu. Technical Report. Nanjing, Southeast University. 
[9] Yuan, H. Y., Liu, F. H. \& Zhang, K. (2008). The Key Skill of Development and Research on Intelligent Transportation Systems (ITS) Architecture in China. Technology \& Economy in Areas of Communications, 10 (4), 88-90.

[10] Zhang, K., et al. (2007). Development of Regional ITS Architecture for Jiangsu Province. Journal of Transportation Systems Engineering and Information Technology, 7 (2): 141-146.

[11] Hughey, D. (2009). Comparing Traditional Systems Analysis and Design with Agile Methodologies. University of Missouri St. Louis. Retrieved 11 August, 2015, from http://www.umsl.edu/ hugheyd/is6840/introduction.html.

[12] Parnas, D. L., Clements, P. C. (1986). A rational design process:
How and why to fake it. IEEE Transactions Software Engineering, 12 (1): 251-257.

[13] McConnell, S. (2004). Code Complete, 2nd edition. Microsoft Press, ISBN 1-55615-484-4.

[14] Boehm, B. (1986). A Spiral Model of Software Development and Enhancement. ACM SIGSOFT Software Engineering Notes, ACM, 11 (4): 14-24.

[15] Boehm, B. (1988). A Spiral Model of Software Development and Enhancement. IEEE Computer, IEEE, 21 (5): 61-72.

[16] Padmadas, M. et al. (2010). A Deployable Architecture of Intelligent Transportation System - A Developing Country Perspective. IEEE International Conference on Computational Intelligence \& Computing Research, Tirunelveli, India. 